UNIVERSITÄTSBIBLIOTHEK BRAUNSCHWEIG

Tobias Carsten Müller, Olaf Krieger, Andreas Breuer, Klaus Lange, Thomas Form

A Heuristic Approach for Offboard-Diagnostics in Advanced Automotive Systems

Braunschw eig : Inst. Für Regelungstechnik. - Wolfsburg : Volksw agen AG, 2009

Veröffentlicht: 25.09.2009

http://www.digibib.tu-bs. de/?docid=00030354 


\title{
A Heuristic Approach for Offboard-Diagnostics in Advanced Automotive Systems
}

\author{
Tobias Carsten Müller \\ Technische Universität Braunschweig, Institute of Control Engineering \\ Olaf Krieger, Andreas Breuer, Klaus Lange, Thomas Form \\ Volkswagen AG
}

\begin{abstract}
As the complexity of current automobiles increases, new and innovative diagnostic methods for car maintenance and diagnostic inspection are greatly needed. This paper introduces a new diagnostic approach, which learns from previous repair cases with the help of neural networks in order to assist future diagnostic inspections. Practical experiments have shown that this approach is able to provide promising results even with the data that is already available today.
\end{abstract}

\section{INTRODUCTION}

A great challenge in the development of new vehicles consists of improving their reliability and maintainability despite the increasing complexity [1]. As a result, innovative diagnostic methods and systems are needed. Many causes of system failures in highly networked and distributed systems such as today's automobile electronics can only be identified reliably with consistent and system-overlapping diagnostic concepts. As a result of this, the functional dependencies between individual systems and the networking of control units create multiple interdependencies between these systems [2]. The consequences of errors in such distributed systems are difficult to survey.

Consequently, diagnostic inspections carried out in car repair shops are not able to identify malfunctions in over sixty per cent of the known cases [4]. Therefore, inspection time for repairing as well as replacing functional parts increases. The result is rising costs. Additionally, diagnosis is aggravated by the fact that one symptom can have numerous causes, and one cause often results in several symptoms [3].

\section{STARTING POINT}

Most of today's electronic control units $\left(\mathrm{ECUs}^{1}\right)$ are selfdiagnosable thanks to diagnostic routines within the ECU software. This allows the status estimation of the sensors and actuators involved. Model-based diagnostic methods are often used for that purpose [6]. If a malfunction is recognized by the system, a diagnostic trouble code $\left(\mathrm{DTC}^{2}\right)$ is determined and can be accessed via a diagnostic interface [5].

In contrast to the on-board diagnostics, off-board systems are attached to a vehicle in car repair shops which access the vehicle's systems via a diagnostic interface. These systems provide the possibility to collect the stored diagnostic trouble codes of all available vehicle ECUs.

Efficient diagnostic systems shall not only provide all information from the analyzed ECUs to the customer service employee but also actively support him in fault diagnostics. The guided diagnostic process (GDP) in the customer service of Volkswagen is an example of such systems. The GDP uses inspection plans which are based on decision trees created by experts. These in-

\footnotetext{
${ }^{1}$ An electronic control unit can also be considered a system.

${ }^{2}$ DTC - Diagnostic Trouble Code
} 
spection plans are translated into different languages and distributed to users worldwide. An inspection plan needs to be developed for every single diagnostic trouble code that can occur in a vehicle. Since vehicles can have numerous equipment options it is quite demanding to cover all versions. This fact has to be taken into account when inspection plans are created since malfunctions can have different reasons due to various car models. Therefore, inspection and repair instructions can vary significantly. An additional problem of manually created inspection plans is the difficulty in recognizing and covering all potential malfunction sources in advance.

In practice, the points mentioned above lead inevitably to tradeoffs since taking all possible error sources as well as all vehicle variants into account is only manageable with tremendous effort. Particularly the increasing complexity and the trend towards distributed functionality leads to reduced and more unspecific inspection plans (e.g. "error tracing using circuit diagrams").

An important step towards improving diagnostic quality is to provide capabilities for evaluating events in the repair shops, such as the introduction of a database for repair case data. That way, all relevant data for a repair case, e.g. diagnostic trouble codes, could be recorded. With today's available information, evaluations are already carried out in order to improve the inspection plans. However, this evaluation process as well as the subsequent improvement of the inspection plans are carried out manually and cannot be automated with acceptable effort. Hence, only a low number of inspection plans can be improved by the described procedure. At this point, the experience-based diagnostic system described in this paper is a promising approach.

\section{PRINCIPLE}

There are several thousands of car repair shops for many automobile manufacturers worldwide with more than one hundred thousand employees. The exchange of experience between the individual car repair shops is quite a challenge.

An example illustrates the principle. When a complex repair problem appears, customer service employee 'A' needs a certain amount of time for fault diagnostics as well as for replacing several suspicious parts. That means that customer service employee ' $B$ ' at another car repair shop with the same problem needs to invest the same effort in order to locate the error. Consequently, customer service employee ' $B$ ' does not benefit from the experience of customer service employee ' $A$ ' and vice versa. If previous experiences were to be made available to employee $B$, a potentially large effort reduction could be achieved.

Therefore, the objective should be to learn from past repair cases and to apply the extracted knowledge to future instances. However, similar to the GDP, the resulting amount of data and knowledge is enormous.
Thus, the learning process as well as the application can only be profitably efficient when the complete process is automated.

\section{PROBLEM ANALYSIS}

For a learning system, the type and the quality of the existing data is of decisive importance. The system should learn from previous repair cases in car repair shops. Therefore, the focus should be set on defining a general diagnostic and repairing process first. Figure 1 illustrates this process in simplified form as an iterative process.

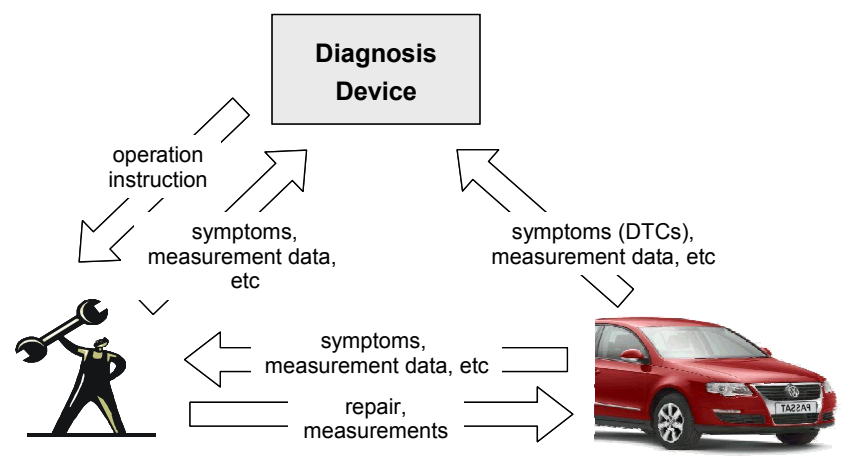

Figure 1: Simplified repair process according to [8]

In the first step, the diagnostic system acquires symptoms which are either taken from the vehicle or entered manually by the user. As shown in Figure 2, such symptoms can be noises, vibrations, measurements, defective functions, diagnostic trouble codes as well as subjectively perceived symptoms that were coded in a suitable way. In the second step, the diagnostic system outputs instructions to the user. These instructions can either be repair actions or possible causes of the fault that the system suggests for inspection.

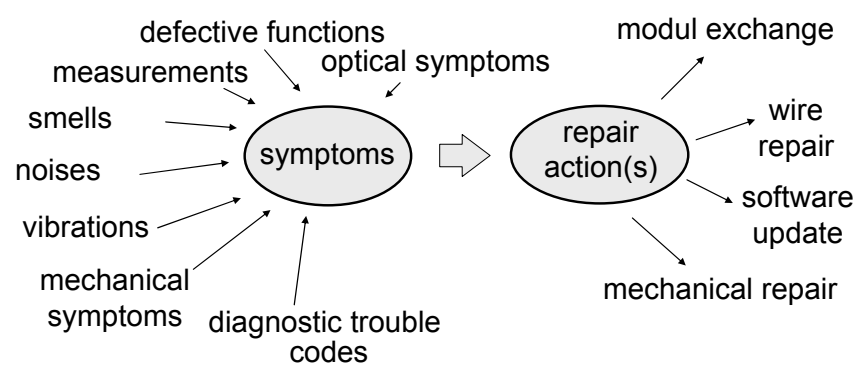

Figure 2: Repair process as a mapping from symptoms to repair actions.

If the suggested instruction did not lead to the identification of the cause of the fault or to the elimination of it, the user will give feedback to the system which will provide further instructions analogously to Step 2. User feedback demanded by the system can be measurements, observations and additional symptoms. This process ends either with a repair that is assumed to be successful by the user or with an undetectable defect in the most inauspicious case. 
The description "No Trouble Found" (NTF) indicates that the cause of the fault was not found by a direct or indirect indication of the diagnostic system. In this case, the diagnostic system was not helpful. The process is superimposed by an iterative process. If the defect was only solved putatively, it is assumed that the customer will return (at least in case of warranty) to the car repair shop in order to request another repair.

According to the repair process described above, the information content of a single repair case Rep according to (1) can be abstracted as a mapping from the repair context $R$ and the symptoms $S_{R}$ to a set of repair actions $A_{R}$ (compare Figure 2). The symptoms $S_{R}$ of the power set $\wp(S)$ of all known symptoms $S$ and the repair actions $A_{R}$ are from the power set $\wp(A)$ of all repair actions $A$. Therefore, a concrete repair case represents a mapping function according to (2).

$$
\begin{gathered}
\operatorname{Rep}: R \times \wp(S) \rightarrow \wp(A) \\
\operatorname{Rep}\left(r, S_{R}\right)=A_{R} \in \wp(A), \text { where } r \in R, S_{R} \in \wp(S)
\end{gathered}
$$

All symptoms $s \in S$ observed during a repair process are summarized in a set of symptoms $s \in \wp(S)$. These symptoms not only include the technically and well-defined symptoms, e.g. DTCs, but also the subjectively perceived symptoms that were coded in an appropriate way. Operations carried out by customer service employees, e.g. exchanged parts or work items, are summarized in the repair actions. Among other things, the repair context $R$ includes general repair-specific attributes like the current date, car repair shop and repair progress which influence the result of the repair process function $R e p$. In this way, a repair can be regarded as a closed process. The system $\Sigma$ which the process $R e p$ is applied to is given by the specific vehicle being observed. However, vehicles to be repaired are not absolutely identically constructed ones. but rather all vehicles are different due to possible variants even if they belong to the same class. Figure 3 illustrates these system variants in accordance with [9].

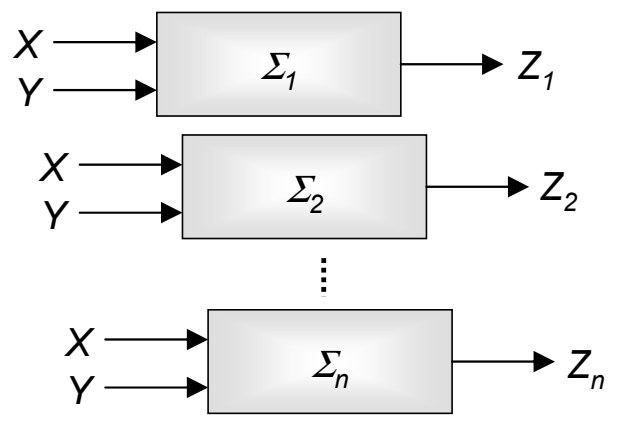

Figure 3: Different system variants.

All observable input values are depicted by the vector $X$. The vector $Y$ contains unobservable input values, while $Z_{i}, 1 \leq i \leq n$ represents the vector of output values. In the following, the variance of a system ${ }^{3} \Sigma_{i}$ is summarized as the system context $C_{i}$. If all observable values $C_{o, i}$ of $C_{i}$ are now considered as additional components of $X$, and all unobservable values $C_{n o, i}$ are regarded as additional components of $Y$, the systems $\Sigma_{i}, 1 \leq i \leq n$ can be assumed to be one resultant system $\Sigma$. Hence, the diversity of variants can also be regarded as part of the learning task. As a consequence, the mapping of (1) to (3) as well as the mapping of (2) to (4) must be extended.

$$
\begin{aligned}
& \operatorname{Rep}: R \times \wp(C) \times \wp(S) \rightarrow \wp(A) \\
& \operatorname{Rep}\left(r, C_{R}, S_{R}\right)=A_{R} \in \wp(A), \\
& \text { where } r \in R, C_{R} \in \wp(C), S_{R} \in \wp(S)
\end{aligned}
$$

The resulting learning task consists of deriving a model from the existing data which reflects the observed input and output values in the best possible way. Only observable values $X$ and $C_{o}$ can be used for modeling which yields to a model $\Pi$ mapping the observable context $C_{o}$ and a subset of the symptoms to a set of repair actions according to (5). For further considerations, $C_{n o}$ will be neglected and, additionally, $C$ will be used instead of $C_{0}$.

$$
\Pi: C_{b} \times \wp(S) \rightarrow \wp(A)
$$

The examination of different technologies at the Institute of Control Engineering, Technische Universität Braunschweig, has shown that artificial neural networks (ANN) are particularly suitable for that purpose. An introduction to ANNs can be found in [7]. An important benefit of ANNs for this particular task is the recognition of patterns within the existing training data. Furthermore, they have the generalization ability enabling them to provide a meaningful result even over symptom sets which are not part of the trained patterns.

\section{IMPLEMENTATION}

The purpose of the realization is a distributed iterative diagnostic approach which permits an automatic feedback taking former earlier experiences into consideration. The principal data flow is shown in the diagram of Figure 4. All processes in the car repair shop are summarized in the repair process. A selection of the resulting data, primarily repair case data according to (3) and (4) respectively, has to be transferred to the data warehouse.

Subsequently, the learning system creates models by using the vast amount of data available in the data warehouse. The resulting models have to be transferred as diagnostic knowledge into a database called a Diagnostic Knowledge Base. This knowledge base will be distrib-

\footnotetext{
${ }^{3}$ The variance of a system can also be regarded as vehicle specific qualities.
} 
uted to the car repair shops. Therefore, it is a process whose feedback improves future quality. As a result, an automatic, iterative problem solving process would arise for not yet known repair problems based on the set of all customer service employees. In the following, this process in described in detail.

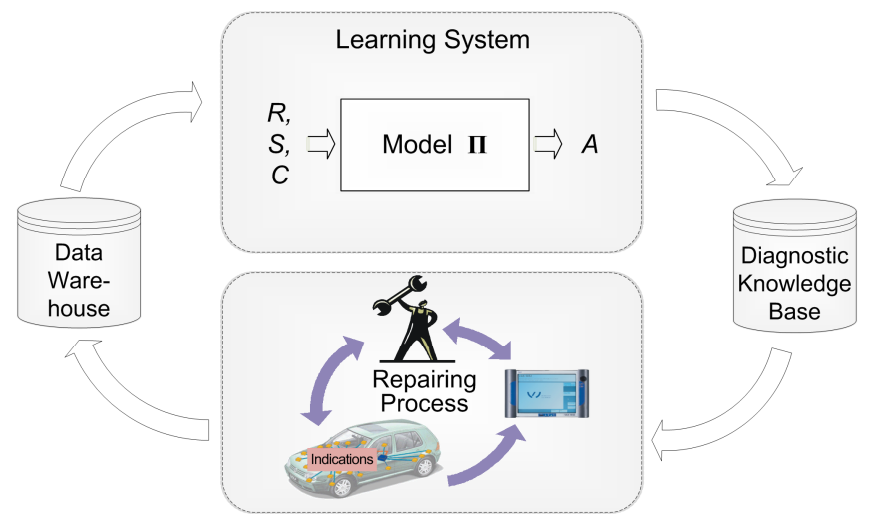

Figure 4: Data flow of the diagnostic process.

If a new repair problem is not yet known to the data warehouse and occurs in a car repair shop, the trained models do not have any diagnostic knowledge for this first appearance. Thus, suitable diagnostics would be impossible. The corresponding customer service employee would have to solve the problem with the help of the available methods, e.g. inspection plans. Furthermore, it is assumed that more than one (also unsuccessful) repair action is carried out during this first repair. These results will then be inserted into the database as well. In the following step, the learning system would again learn from the data stored in the data warehouse and optimize the diagnostic knowledge. If this repair problem appears again in a different car repair shop, the diagnostic system could already perform a more suitable diagnosis due to the updated models. This diagnosis, however, could be unclear due to the data situation. Therefore, the corresponding customer service employee will normally have to choose one of the suggested diagnoses. If the repair was part of the diagnoses, the set of unsuccessful repair actions would become smaller on average. After a couple of iterations the best repair for the new repair problem would be found automatically. According to the same functional principle, not only new repair problem solutions would be found in this manner but also modifications would be adapted automatically.

\section{THE LEARNING SYSTEM}

The learning system plays a decisive role in the diagnostics process. A realization of a learning system with artificial neural networks (ANNs) is shown in Figure 5.

A large feed-forward-network with an optional hidden layer is constructed. The number of input neurons corresponds to the size of the input vector (number of possible symptoms in $S$ and context information $C$ ). The number of output neurons corresponds to the size of the output vector (number of possible repair actions in $A$ ).

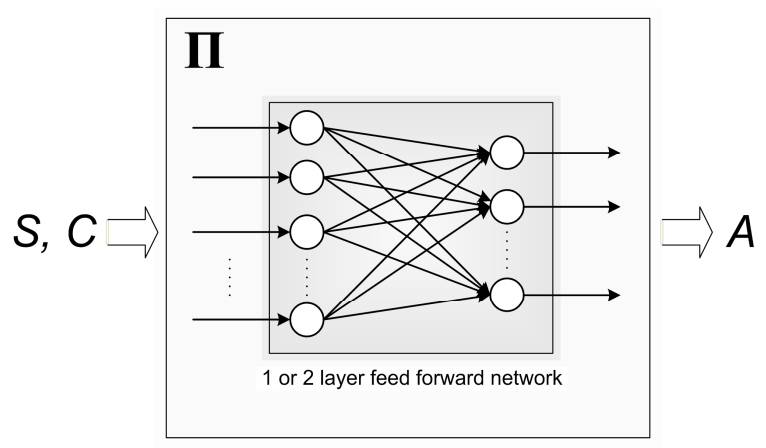

Figure 5: Learning system consisting of one neural network.

When realizing such a system, it has turned out that incomputable large networks arise in the average case. Even if only DTCs are used as symptoms and replacement with spare parts as repair actions, a network with at least 3,000 input and 10,000 output neurons arises for a medium-sized vehicle. The resulting number of connections is between $30,000,000$ and 50,000,000, depending on the number of hidden layers. Due to this high number of connections it is unsuitable to create and train such a net efficiently. In addition, one repair action mostly depends on only a few symptoms. Therefore, the greater part of the connections to an output neuron has a weight close to zero. The large number of irrelevant connections indeed leads to a loss of network quality. It is more appropriate to create smaller and better manageable networks. Hence, the resulting model $\Pi$ should be decomposed into smaller individual models $\Pi_{i}, 1 \leq i \leq n$ as well as to suitably select and merge functions, as shown in Figure 6.

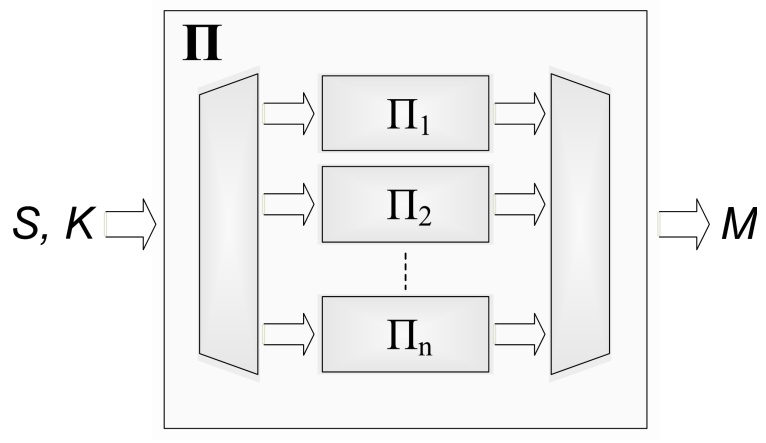

Figure 6: Model composition of individual models.

The model $\Pi$ is computed according to (6) in which Select and Merge are defined according to (7) and (8).

$$
\begin{gathered}
\Pi @ \operatorname{Merge}\left(\Pi_{1}\left(\operatorname{Select}_{1}(S, C)\right), \mathrm{K}, \Pi_{n}\left(\operatorname{Select}_{n}(S, C)\right)\right) \\
\text { Select }_{i}: \wp(S) \times \wp(C) \rightarrow \wp\left(S_{i}\right) \times \wp\left(C_{i}\right) \\
\text { where } S_{i} \subseteq S, C_{i} \subseteq C
\end{gathered}
$$




$$
\text { Merge }_{i}: \wp\left(A_{1}\right) \times \ldots \times \wp\left(A_{n}\right) \rightarrow \wp(A), \text { where } A_{i} \subseteq A
$$

Each submodel consists of a specific neural network. The way in which the set of symptoms and the set of repair actions $S_{i}$ and $M_{i}$ are cut is a complicated problem. In order to retain the benefits of neural networks, namely pattern recognition and generalization, the symptoms and repair actions should be subsumed which are correlated to each other. A measure for the correlation is the statistical significance. Significance describes the existence of a correlation between a symptom and a repair action with a certain probability $p$ of error (called a p-value). While a $p$-value of 1 means a hundred percent probability of error for the correlation (hence, there is no correlation), a $p$-value of 0 means a 0 percent probability of error (there is strong correlation).

\section{CLUSTERING ARTIFICIAL NEURAL NETWORKS}

A special clustering method was developed (compare [8] Section 7) for appropriate partitioning into smaller networks. However, further evaluations have shown that this method has several disadvantages especially for larger amounts of data.

The procedure tends to produce huge clusters with an increasing amount of data. Although this can be diminished by the introduced compensation method, the quality of the clusters also degrades. This approach was consequently improved in order to eliminate the described disadvantages and will be discussed in the following.

According to the previous approach, the first step is locating significant symptoms for each repair action. Potential methods for such a significance test are the $\chi^{2}$ Test (Chi-Square Test) according to (9) as well as the Fisher's exact test according to [12] to name 2 examples. If such a significance test is carried out for the entire database with all symptoms and repair actions successively, it yields the significance matrix $S$ (11). The influence of symptoms on the repair actions can be retrieved from the significance matrix based on the probability of error ( $p$-value). The $p$-value is calculated from the test statistic $\chi^{2}$ using the approximation formula (10).

In the second step, the significance matrix $S$ is converted into the distance matrix $D$ (compare (12)). For each repair action and symptom, the distance $d$ from a symptom to a repair action is computed. For that purpose, the individual distances ( $p$-values) between repair actions and symptoms are represented as a bipartite, non-directional, weighted graph $G$ according to (13). The node set (14) results in a disjoint/real partitioning into repair action nodes $V_{A}$ and symptom nodes $V_{S}$. According to (15), the edges $E$ are enriched by the edge weights $p_{s, m}$ for the edges $e_{s, m} \in E$ from the significance matrix $S$.

$$
\begin{gathered}
\chi^{2}=\sum_{\mathrm{j}=1}^{u} \sum_{\mathrm{k}=1}^{\mathrm{r}} \frac{\left(\mathbf{n}_{\mathrm{jk}}-\mathbf{n}_{\mathrm{jk}}^{*}\right)^{2}}{\mathbf{n}_{\mathrm{jk}}^{*}} \\
\mathbf{p}=\frac{1}{\mathbf{2}} \cdot \mathbf{1 0}-\frac{\chi^{2}}{3,84} \\
\mathbf{S}=\left[\begin{array}{ccc}
\mathbf{p}_{\mathrm{a}_{1} \mathrm{~s}_{1}} & \ldots & \mathbf{p}_{\mathrm{a}_{1} \mathrm{~s}_{\mathrm{i}}} \\
\vdots & \ddots & \vdots \\
\mathbf{p}_{\mathrm{a}_{\mathrm{j}} \mathrm{s}_{1}} & \ldots & \mathbf{p}_{\mathrm{a}_{\mathrm{s}_{\mathrm{s}}}}
\end{array}\right]
\end{gathered}
$$

$$
\begin{aligned}
\chi^{2} & =\text { test statistic for the } \chi^{2}-\text { test } \\
\mathbf{n}_{\mathrm{jk}} & =\text { marginal totals } \\
\mathbf{n}_{\mathrm{jk}}^{*} & =\text { expected marginal total for } \mathbf{H}_{0} \\
\mathbf{u}, \mathbf{r} & =\text { attribute dimension (here 2) }
\end{aligned}
$$

The shortest path between repair action $a_{i}$ and symptom $s_{j}$ is now given as the edge set (16). Hence, the sum of the edge weights for the shortest path between $a_{i}$ and $s_{j}$ is obtained according to (17). An example for repair action $a_{1}$ and symptom $s_{8}{ }^{4}$ is shown in Figure 7 .

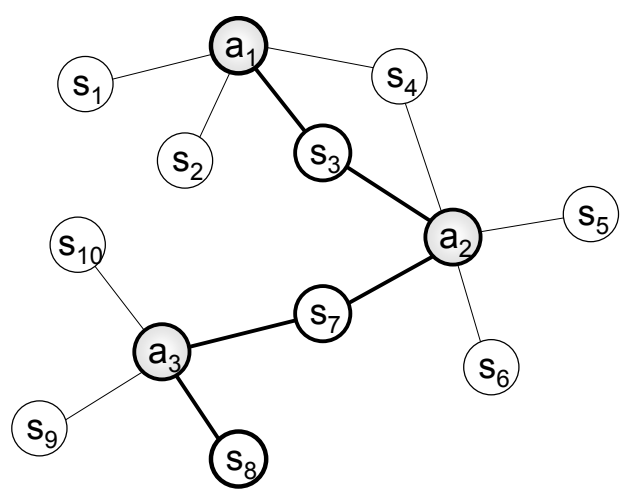

Figure 7: Illustration of distance between symptom $s_{8}$ and repair action $a_{1}^{5}$.

The corresponding equation is defined with (18) and is not arranged according to the matrix $S$ (for demonstration purposes).

The necessity to choose paths via repair action nodes results from the distribution of the repair case data. Typical probabilities for the appearance of an arbitrary symptom $s_{x}$ in a repair case lie in the range of $0.02 \% \leq \mathrm{P}\left(s_{x} \mid F\right) \leq 0.2 \%$, assuming that a fault $F$ is present. As a result, positive correlations can be obtained easily, while negative correlations cannot be determined. In this case, under consideration for the $\chi^{2}$ Test according to (9) we have two variables with two features at once: symptom available $\left(h_{x 1}\right) /$ not available $\left(h_{x 2}\right)$; repair action carried out $\left(h_{1 x}\right) /$ not carried out $\left(h_{2 x}\right)$.

\footnotetext{
${ }^{4} s_{8}$ represents symptom $8, a_{1}$ represents repair action 1 .

${ }^{5}$ Symptom $s_{8}$ represents symptom 8 , repair action $a_{1}$ represents repair action 1 . The numeration for the demonstration is chosen arbitrarily.
} 
Thus, the $\chi^{2}$-Test can be converted and simplified according to (19). This special case is called $2 \times 2$ contingency table.

$$
\begin{aligned}
& \mathbf{D}=\left[\begin{array}{ccc}
\mathbf{d}_{a_{1} s_{1}} & \ldots & \mathbf{d}_{\mathbf{a}_{1} \mathbf{s}_{1}} \\
\vdots & \ddots & \vdots \\
\mathbf{d}_{a_{j_{1}} s_{1}} & \ldots & \mathbf{d}_{a_{j} s_{1}}
\end{array}\right] \\
& \mathbf{G}=(\mathbf{V}, \mathbf{E}) \\
& \mathbf{V}=\mathbf{V}_{\mathrm{S}} \cup \mathbf{V}_{\mathrm{A}} \\
& \mathbf{E} \subseteq \mathbf{V}_{\mathrm{A}} \times \mathbf{V}_{\mathrm{S}} \\
& K_{A_{i}, s_{j}}=\bigcup_{k=1 . . n}\left\{\begin{aligned}
e_{k} \in E \mid e_{1} & =\left(a_{i}, s_{1}\right), e_{2}, \ldots, e_{n-1}, e_{n} \\
& =\left(a_{n}, s_{j}\right)
\end{aligned}\right\} \\
& \mathbf{d}_{\mathrm{a}_{\mathrm{i}} \mathrm{s}_{\mathrm{j}}}=\sum_{\mathrm{e}_{\mathrm{a}, \mathrm{s}} \in \mathrm{K}_{\mathrm{A}_{\mathrm{i}}, \mathrm{s}_{\mathrm{j}}}} \mathbf{p}_{\mathrm{a}, \mathrm{s}} \\
& \mathbf{d}_{\mathrm{a}_{1} \mathrm{~s}_{8}}=\mathbf{p}_{\mathrm{a}_{1} \mathrm{~s}_{3}}+\mathbf{p}_{\mathrm{s}_{3} \mathrm{a}_{2}}+\mathbf{p}_{\mathrm{a}_{2} \mathrm{~s}_{7}}+\mathbf{p}_{\mathrm{s}_{7} \mathrm{a}_{3}}+\mathbf{p}_{\mathrm{a}_{3} \mathrm{~s}_{8}}
\end{aligned}
$$

The $\chi^{2}$-Test statistic, however, is inappropriate for an illustrative comparison and is converted with respect to (20) into the Phi-coefficient (21). Unlike the $\chi^{2}$-Test statistic, the Phi-coefficient represents a normalized measure (also called correlation) within the domain of -1 $\leq \Phi \leq 1$. As $\Phi>0$ indicates a positive correlation, a $\Phi<0$ stands for a negative correlation. For the maximum or minimum of the correlation, the entries of the main diagonal or secondary diagonals of $H$ must be zero. This can be easily accomplished for a positive correlation since the entries $h_{12}$ and $h_{21}$ must be equal to zero. Furthermore, the conditionally high valued $h_{22}$ resulting from the repair case data affects these two entries $h_{12}$ and $h_{21}$ carrying less impact. For a negative correlation, however, the entries $h_{11}$ and $h_{22}$ have to be equal or close to zero, which is impossible for $h_{22}$. The determination of the extremum of (21) yields the fact that the strongest negative correlation is reached under the condition $h 12=h 21$. Therefore, the minimum of $\Phi$ can be computed to (22).

The range of $\Phi_{\min }$ can be determined for the previously mentioned typical appearance probabilities $\mathrm{P}\left(s_{x} \mid F\right)$ to $10^{-4} \leq \Phi_{\min } \leq 10^{-3}$. Hence, it is obvious why negative correlations can only be recognized with difficulty or cannot be recognized at all. Under the assumption that such a negative correlation represents a positive correlation for another repair action, taking a path via another repair action is a solution of this problem.

The generated distance matrix $D$ as defined in (12) provides the basis for the cluster generation in Step 3 . Whereas the method introduced in [8] not only combines several symptoms but also several repair actions in one cluster only one repair action per cluster exists in our method. In this special case, it can be shown that the generalization of a neural network is not decreased by the separation into individual networks (one for each output neuron). It is not necessary to combine more than one repair action within one cluster. This leads to results with the advantage that the individual networks become more clearly arranged as well as more robust, which particularly applies to the use containing hidden neurons ${ }^{6}$. In case of a too small number of hidden neurons in combination with uncorrelated output neurons, unintentional side effects can arise which intensively reduce the generalization performance.

$$
\begin{gathered}
\chi^{2}=\frac{n \cdot\left(h_{11} h_{22}-h_{12} h_{21}\right)^{2}}{\left(h_{11}+h_{12}\right) \cdot\left(h_{21}+h_{22}\right) \cdot\left(h_{11}+h_{21}\right) \cdot\left(h_{12}+h_{22}\right)} \\
\chi^{2}=\mathbf{n} \cdot \Phi^{2} \\
\sqrt{\left(h_{11}+h_{12}\right) \cdot\left(h_{21}+h_{22}\right) \cdot\left(h_{11}+h_{21}\right) \cdot\left(h_{12}+h_{22}\right)} \\
\Phi_{\min }=-\frac{h_{12} h_{22}-h_{12} h_{21}}{h_{12}+h_{22}} \quad \text { für } \quad h_{12}=h_{21} \text { und } h_{11}=0 \\
H=\left[\begin{array}{ll}
h_{11} & h_{12} \\
h_{21} & h_{22}
\end{array}\right]=\text { contingency table } \\
n=\text { sample size }
\end{gathered}
$$

The core of every cluster $C_{m i}$ therefore belongs to a single repair action $a_{i}$. By a previously defined threshold distance $d_{t h}$, all those symptoms whose distance is below the threshold distance $d_{t h}$ are added to the cluster $C_{m i}$ according to (23). This is shown in Figure 8.

$$
\mathbf{C}_{\mathrm{a}_{\mathrm{i}}}=\bigcup_{\mathrm{s} \in \mathrm{V}_{\mathrm{s}}}\left\{\mathbf{d}_{\mathrm{a}_{\mathrm{i}}} \leq \mathbf{d}_{\mathrm{th}}\right\}
$$

$$
C_{A_{i}}=\text { cluster for repair action } A_{i}
$$

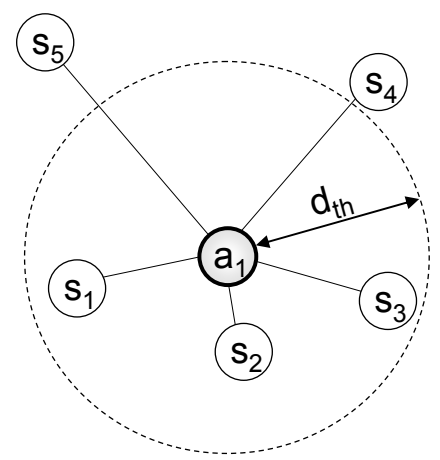

Figure 8: Exemplary depicted distances and the distance threshold $d_{t h}$ for repair action $a_{1}$.

\footnotetext{
${ }^{6}$ Neurons in a hidden layer are also called hidden neurons.
} 
Using the definition of the $\chi^{2}$-Test (refer to (9) and [11]), the individual $p$-values according to (10) as well as the distance values $d_{i j} \in D$ according to (17) depend on the sample size $n$. However, for the determination of the parameter $d_{t h}$, it is desirable that this parameter should not be dependent on the sample size but rather be normalized. The relation of the $\chi^{2}$-Test to the phicoefficient is contemplated in (20). Unlike the $\chi^{2}$-Test, the phi-coefficient represents a normalized measure of correlation $^{7}$ with the range of $-1 \leq \Phi \leq 1$. A sample size independent distance threshold $d_{t h, n o r m}$ is consequently reached if the distance $d_{t h}$ needed for computation is defined according to (24) based on (9), (10), and (20). By summation of the individual distances in the graph, $d_{\text {th,norm }}$ can also reach a value $>1$.

$$
\begin{aligned}
& d_{\text {th }}=\frac{1}{2} \cdot 10^{\frac{n \cdot d_{\text {th,norm }}^{2}}{3,84}} \\
& n=\text { sample size }
\end{aligned}
$$

After the cluster generation step, an ANN is created in Step 4 for each cluster, where every symptom is represented by an input neuron, and every repair action is represented by an output neuron. The neurons are connected feed-forward in the same manner as the previous realization, which is the classic configuration of a pattern associator [10]. Hidden layers can optionally be inserted. Due to the previous evaluation of exemplary data, the sigmoid function according to [7] turned out to be particularly advantageous for the activation function.

\section{EVALUATION}

Several test cases with data sets from real repair cases could already be carried out with a modular training and evaluation software. A particularly difficult task was the acquisition of the data. Furthermore, the acquisition as well as the integration of the data sets was time-consuming. It has to be mentioned that the data generation process in the car repair shops does not have the desirable quality for the learning system. Only with an extensive effort was it possible to preprocess and merge the data sets and further develop an algorithm which preferentially selects "high-quality" data sets.

The starting point for the test cases was a large number of real repair cases. DTCs were used as symptoms, while spare parts, work items and component names ${ }^{8}$ served as repair actions. With the help of the training software, neural networks were created, trained and subsequently validated using the existing data. Different validation and evaluation methods are discussed in [14]. Moreover, an experimental application software was developed in order to test the diagnostic performance

\footnotetext{
${ }^{7}$ Here, the meaning of correlation is not the correlation known from staistics.

${ }^{8}$ Component names $=$ A catalog, established for statistical evaluations, with repair actions which are each assigned to one component, e.g. oil level sensor.
}

of the trained neural networks directly with the vehicle. A screenshot of the application software is shown in Figure 9.

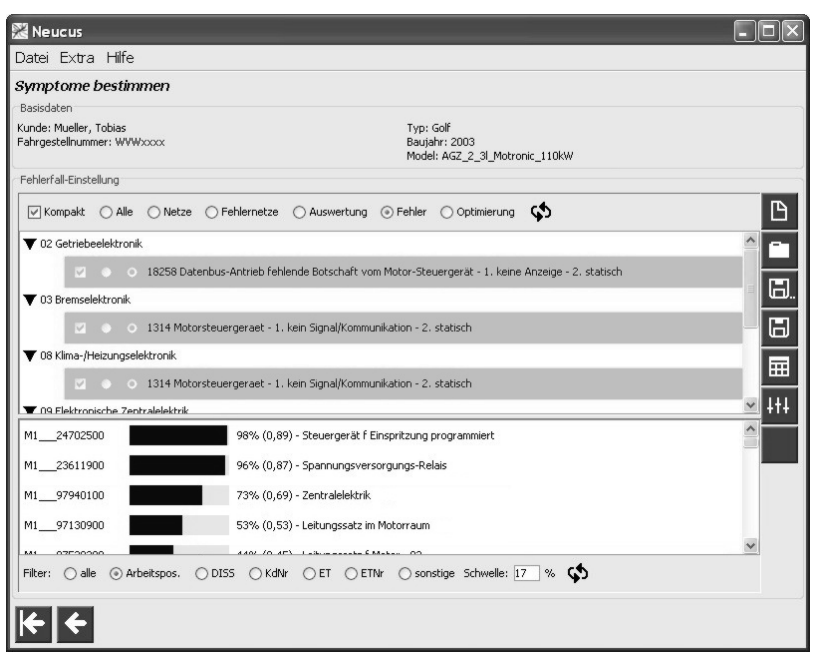

Figure 9: Screenshot of the application software [13].

For additional evaluation purposes the system was applied to a real vehicle. Several faults were injected into the vehicle in order to evaluate the repair actions suggested by the diagnostic system. For every fault the system provided a suitable solution within the first five suggestions.

Beside manual evaluation there is an additional method to determine the performance in an automatic way, described in [14] but the method requires an unambiguous and faultless database for precise performance determination. This requirement is not fulfilled by the existing data. Nevertheless the automatic evaluation is enormously helpful for the development process although it does not yield an exact performance value. In order to determine such a value, another manual evaluation was conducted alongside the already described manual evaluation of a real vehicle. The evaluation is based on 100 reference cases randomly chosen from the data warehouse. For each case, the repair actions were compared to those suggested by the diagnostic system and those that would have actually led to the solution or would have provided the crucial evidence for the solution.

\begin{tabular}{|l|c|}
\hline & Success rate \\
\hline Hit ranked first & $78 \%$ \\
\hline Hit ranked second & $6 \%$ \\
\hline Hit ranked third & $5 \%$ \\
\hline $\begin{array}{l}\text { Hit ranked fourth or } \\
\text { beyond }\end{array}$ & $5 \%$ \\
\hline Total & $\mathbf{9 4 \%}$ \\
\hline
\end{tabular}

Table 1: Results of the manual evaluation. 
Table 1 presents the results of the manual evaluation regarding the hit rate. $A$ hit ranked first means that the first repair action suggested by the diagnostic system would have yielded the solution of the repair problem (compare Figure 9). It is remarkable that the total hit rate of $94 \%$ is already achieved with the current data acquisition. As previously mentioned, the current data acquisition is conceived primarily for statistical evaluations and for this reason does not have the desired quality of a learning system. By improving the data acquisition the efficiency of the diagnostic system can be considerably increased.

\section{CONCLUSION AND FUTURE WORK}

The implemented evaluations indicate that the neural networks provide good results, not only in theory but also in practice. If the utilized data is considered, it is evident that even with existing unclear data, neural networks recognize patterns and correlations automatically which can be used for practical diagnostics. In addition, the models created with the help of ANNs yield a set of new possibilities to understand and to use the data to improve quality. Furthermore, an experience-based diagnostic system represents an excellent addition to existing, widely used diagnostics systems (e.g. GDP). It has the crucial advantages of automatic generation and adaptation of diagnostic knowledge. However, several steps for an efficient integration into the previous processes are necessary. For instance, the integration of the suggested repair actions with a special inspection plan would be desirable. An excellent approach to the generation of dynamic inspection processes was introduced in [15]. Such diagnostic systems allow the efficient repair of future vehicles with continuingly increasing complexity, numerous variants and reduced development cycles.

\section{ACKNOWLEDGMENTS}

The authors thanks go to Dipl.-Inform. Malte Lochau and Dipl.-Ing. Jörn Marten Wille for helpful discussions.

\section{REFERENCES}

1. Foran, T.; Jackman, B., An intelligent diagnostic system for distributed, muti-ECU automotive control systems. presented at SAE World Congress, 200501-1444, 2005

2. Fröberg, J.; Sandström, K.; Norström, C.; Hansson, $\mathrm{H}$.; Axelsson, J.; Villing, B., Correlating bussines needs and network architectures in automotive applications - a comparative case study. Proceedings of the 5th IFAC Int. Conference on Fieldbus Systems and their Applications (FET). Aveiro, Portugal, 2003
3. A. Unger, K. Lange, D. Peters, H.C. Reuss, Methods of a Holistic System View for Function-Oriented Error Detection and Diagnosis in Automotive Networks. 12. Int. Congress on Electronic Systems for Vehicles, Baden-Baden, Germany, 2005, ISBN 318-091907-8

4. Mohammad Abuosba, 2003. Fahrzeug-Diagnose mit System. Journal Wissensmanagement, 8/03

5. ISO 15031, Road vehicles - Communication between vehicle and external equipment for emmissions-related diagnostics. ISO 2006

6. Isermann, R: Fault-Diagnosis Systems, An Introduction from Fault Detection to Fault Tolerance. Springer Berlin Heidelberg, 2006

7. Bishop, Christopher M., Neural Networks for Pattern Recognition. Oxford University Press, 1995

8. T. Müller, K. Lange, A. Breuer, O. Krieger, T. Form, Automatic and Experience-based Diagnostics using Distributed Data and Neural Networks. 13. Int. Congress on Electronic Systems for Vehicles, Baden-Baden, Germany, 2007, ISBN 978-3-18092000-9

9. Cherkassky, V.; Mulier, F. Learning from Data. Concepts, Theory, and Methods, 2nd Edition, WileyIEEE Press, 2007, ISBN 978-0-471-68182-3

10. Ripley, B.D.: Pattern Recognition and Neural Networks. Cambridge University Press, 1996

11. Rice, John A., Mathematical Statistics And Data Analysis. Duxbury Press, 2006

12. Fisher, R. A., Statistical Methods for research workers. Oliver and Boyd, 1954

13. Wittling, Daniel, Entwicklung und Optimierung von Anwendungs- und Validierungsmethoden für die Fehlerdiagnose mit neuronalen Netzen. Thesis, Technische Universität Braunschweig, Germany, 2008

14. T. Müller, O. Krieger, T. Form, Evaluierung von Offboard-Diagnosesystemen am Beispiel eines erfahrungsbasierten Diagnoseverfahrens. 3rd Conference on Simulation und Test, Berlin, Germany, 2008, ISBN 978-3-8169-2818-8

15. O. Krieger, A. Breuer, T. Müller, T. Form, K. Lange, Wahrscheinlichkeitsbasierte Fahrzeugdiagnose auf Basis individuell generierter Prüfabläufe. Conference on Mechatronik 2007 - Innovative Produktentwicklung, Wiesloch, Germany, 2007, ISBN 978-318-091971-3 